\title{
Management of Anti-vascular Endothelial Growth Factor Therapy - Diagnostic Pitfalls
}

\author{
Martine Mauget-Faÿsse, ${ }^{1}$ Benjamin Wolff and Chrysanthi Basdekidou²
}

1. Centre Ophthalmologique Robelais, Lyon; 2. Foundation A de Rothschild, Paris

\begin{abstract}
Age-related macular degeneration (AMD) is the leading cause of irreversible vision loss in adults aged over 50 years in developed countries. In 2006, the use of intravitreal injections of anti-vascular endothelial growth factor (anti-VEGF) drugs revolutionised the therapeutic approach to treating neovascular AMD. However, it is essential to detect the frequent diagnostic pitfalls encountered in this therapeutic domain to apply anti-VEGF therapy successfully and thereby avoid useless treatments. Classic pitfalls include - retinal pigmented epithelium-photoreceptor abnormalities mimicking choroidal neovascularisation; persistent serous retinal detachment; pseudocysts and cystoid cavities mimicking retinal oedema; and outer retinal tubulations. Current imaging technologies, such as colour fundus photography, autofluorescence (FAF) and infrared imaging, fluorescein (FA) and indocyanine green (ICGA) angiographies, spectral domain optical coherence tomography (SD-OCT) and enhanced depth imaging OCT (EDI-OCT), can help overcome these pitfalls. Even if anti-VEGF treatments are mostly based on SD-OCT data, the consolidation and correlational analysis of all the available patient data is essential for accurate diagnosis.
\end{abstract}

\section{Keywords}

Age-related macular degeneration (AMD), anti-vascular endothelial growth factor (anti-VEGF) drugs, autofluorescence imaging, cystoid cavity, diagnostic pitfalls, intravitreal injections, outer retinal tubulations, persistent serous retinal detachment, pseudocyst, retinal pigmented epithelium bump, spectral-domain optical coherence tomography

Disclosure: Martine Mauget-Faÿsse has served on the advisory board of Novartis. Benjamin Wolff and Chrysanthi Basdekidou have no conflicts of interest to declare. Received: 24 November 2011 Accepted: 15 December 2011 Citation: European Ophthalmic Review, 2012;6(1):51-5 DOI: 10.17925/EOR.2012.06.01.51 Correspondence: Martine Mauget-Faÿsse, 12-14 rue Rabelais, 69003 Lyon, France. E: mauget_faysse@hotmail.com

Age-related macular degeneration (AMD) is the leading cause of irreversible vision loss in adults aged over 50 years in developed countries $^{1,2}$ and, given the ageing of the populations of these countries, its prevalence is likely to increase dramatically over the coming years. ${ }^{3,4}$

Several ocular diseases, including exudative AMD, are characterised by new choroidal vessel formation leading, if untreated, to severe vision loss. Current knowledge of AMD pathogenesis has contributed to the development of new treatment modalities. Vascular endothelial growth factor (VEGF) has a key role in promoting angiogenesis and vascular leakage in exudative AMD. ${ }^{5.6}$ All retinal cells can be damaged, provoking specific intraretinal anomalies that can now be detected owing to the different imaging techniques currently available.

The era of pathway-based therapy for the early and late stages of AMD has begun,? but anti-VEGF drugs in wet AMD, according to the most important randomised clinical trials, ${ }^{8-12}$ are still the only therapeutic modality that can regress neovascularisation. Currently, patients with exudative AMD are treated according to the recommendations of these clinical trials. Thus, the patients benefit from repeated intravitreal injections of anti-VEGF drugs and regular follow-up to evaluate their neovascular activity. The latter is based on interpreting SD-OCT images along with filter photographs, angiography and biomicroscopy findings.
Neovascular formation is also accompanied by an influx of inflammatory cells, followed by fibroblast formation that leads to fibroglial scarring. ${ }^{13}$ This phenomenon can be observed despite the administration of anti-VEGF drugs. Overcoming the various diagnostic pitfalls is undoubtedly the key to arriving at the best therapeutic decision.

\section{Anti-Vascular Endothelial Growth Factor Treatment Guidelines}

Anti-VEGF treatment guidelines are based on the interpretation of high-resolution OCT imaging and on improved angiography imaging thanks to confocal systems and eye trackers. However, interpreting these examinations alone, without taking into account the clinical aspect, can lead to numerous diagnostic mistakes or inaccuracies. Thus, the examination and comparison of these data with the results of clinical examination is essential.

OCT is an indispensable tool for the follow-up and treatment of exudative AMD. By detecting exudative phenomena, it indicates the necessity of treatment with intravitreal anti-VEGFs. OCT signs of neovascular activity comprise - serous retinal detachment; presence of cystoid spaces seen as hyporeflective intraretinal cavities (cysts and pseudocysts); areas of retinal thickening; and the presence of intraretinal hyper-reflective dots. Some of these intraretinal hyporeflective cavities, however, are neither progressive nor sensitive to anti-VEGF treatment. Thus, they can 


\section{Figure 1: Varied Acquired Vitelliform Lesions}
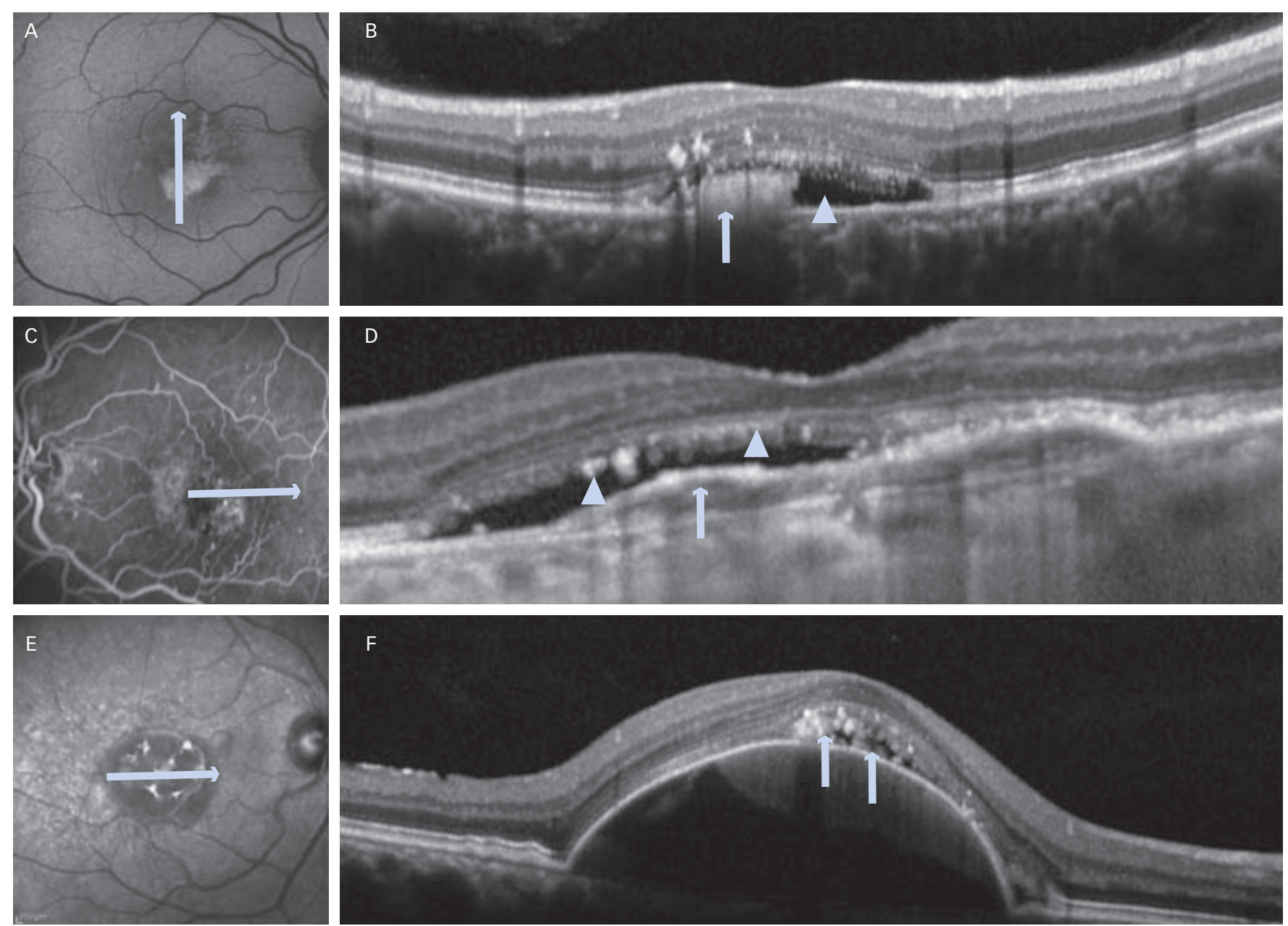

Type 1 - A: fundus autofluorescence (FAF) demonstrates hyperautofluorescence; B: corresponding FAF spectral domain optical coherence tomography (SD-OCT) shows fragmented vitelliform lesion (arrows) and pseudosubretinal fluid (arrow heads) at the superior edge of the acquired vitelliform lesions (AVL). Type $2-C$ : fluorescein angiogram with D, corresponding SDOCT showing hyper-reflective material in the subretinal space (arrow heads) and focal thickening (or bumps) of the retinal pigment epithelium (RPE) band (arrows). Type 3 - E: FAF showing a hyperautofluorescent lesion; F: hyper-reflective material (arrows) in the subretinal space overlying a serous retinal pigment epithelium detachment.

sometimes be used to interpret wrongly a mechanism of chronic retinal degeneration as neovascular activity.

\section{Classic Pitfalls}

Classic pitfalls encountered during anti-VEGF treatment are:

- retinal pigment epithelium (RPE)-photoreceptor abnormalities mimicking active choroidal neovascularisation (CNV);

- persistent serous retinal detachment;

- pseudocysts and cystoid cavities mimicking retinal oedema; and

- outer retinal tubulations.

Pitfall 1 - Retinal Pigment Epithelium-Photoreceptor Abnormalities Mimicking Active Choroidal Neovascularisation

One of the most frequent pitfalls encountered is acquired vitelliform lesions (AVL). ${ }^{14,15}$ These have multiple etiologies and are obvious on fundus autofluorescence imaging (FAF). However, on FA and ICGA they mostly mimic the staining of active occult CNV.

RPE dysfunction results in yellowish deposits in the subretinal space above the RPE band, elevating the overlying neurosensory retina and mimicking CNV. Subretinal fluid can be present in $21 \%$ of AVL cases without the presence of new choroidal vessels. AVL is rarely associated with CNV; in the non-neovascular form, an important sign is the absence of deposits between the RPE and Bruch's membrane, and no exudates or haemorrhages on fundus biomicroscopy. In all cases, SD-OCT identifies AVL as hyper-reflective material in the subretinal space and above the RPE band.

Clinically, three distinct types of AVL can be detected, all mimicking CNV activity:

- Type $1^{14}-\mathrm{AVL}$ can present as a pseudo-serous retinal detachment with vitelliform material (see Figure $1 A$ and $B$ ). Patients might complain of recent metamorphopsia. On FAF, hyperautofluorescent areas are visible that are often associated with hypofluorescent areas of RPE atrophy. FA shows usually hyperfluorescent areas of RPE alterations with diffusion in the late phases mimicking occult CNV. In the early phase of AVL, ICGA shows hypofluorescent spots surrounded by areas of increasing hyperfluorescence but without leakage in the late phases. Retinal maps can show retinal thickening.

It is important to correlate the hyperautofluorescent areas seen in FAF with the OCT scans to identify the subretinal material above 
Figure 2: Chronic Central Serous Chorioretinopathy
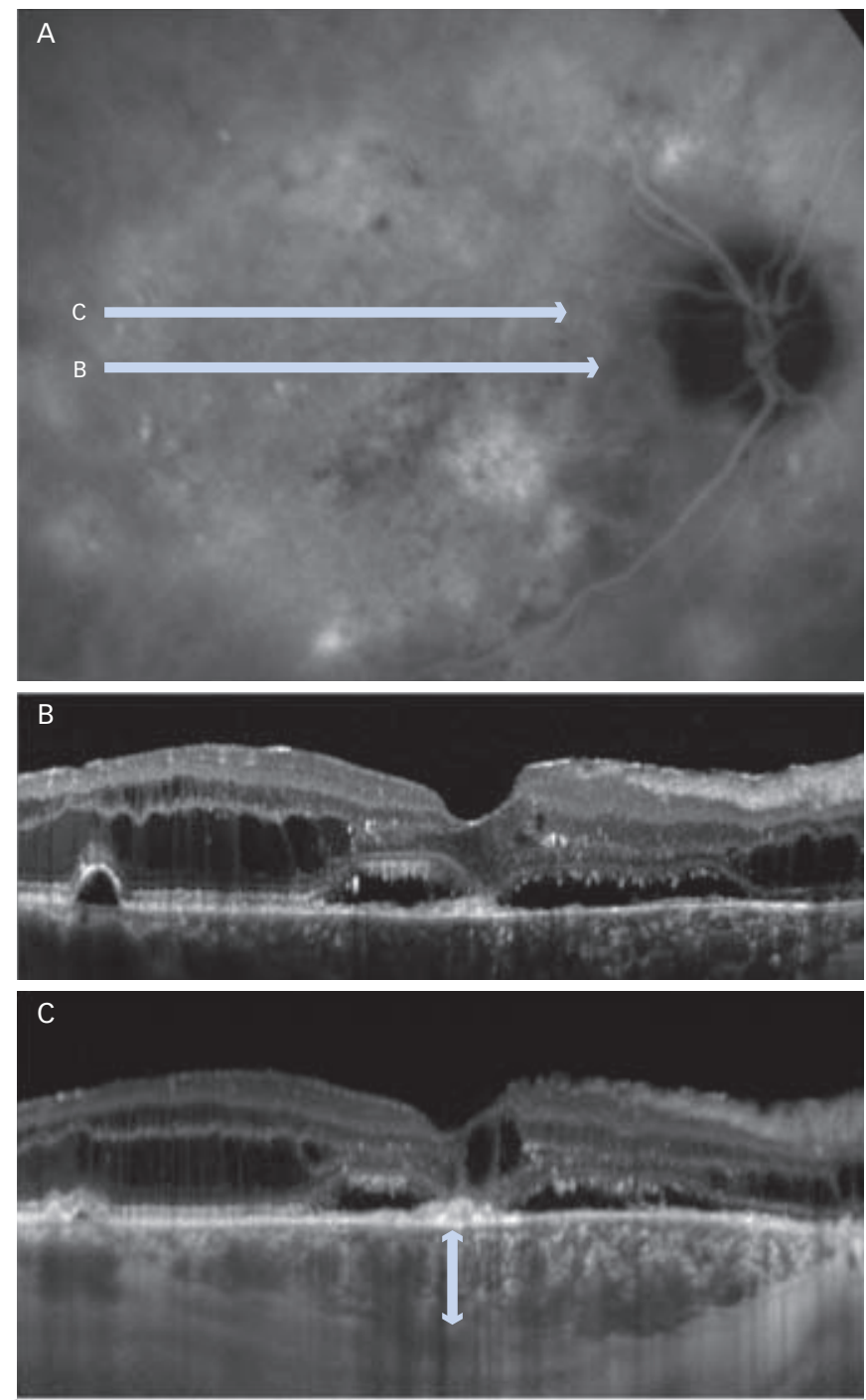

Spectral domain optical coherence tomography (SD-OCT) shows serous retinal fluid, focal thickening of the retinal pigment epithelium (RPE) and cystoid cavities (B). Enhanced depth imaging (EDI)-OCT demonstrates increased choroidal thickness (arrow) (C). Corresponding indocyanine green angiography (ICGA) demonstrates inner choroidal staining in the midstage of angiogram (A).

\section{Figure 3: Retinal Pseudocysts}

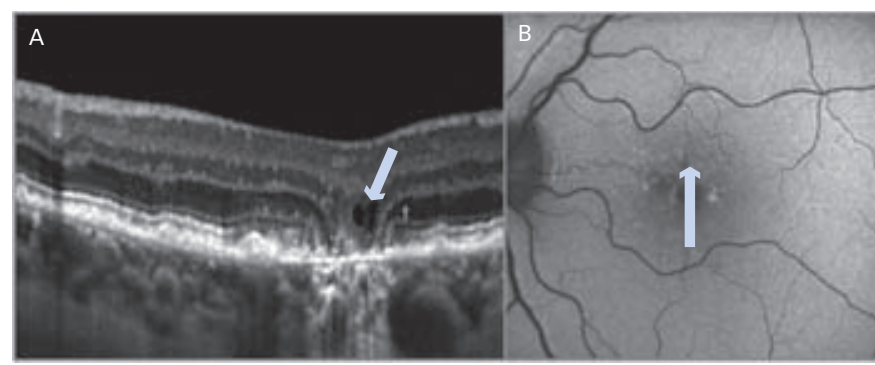

Retinal pseudocysts (here after drusen resorption) (arrow) are located in the inner nuclear layer (A) above a hypoautofluorescent retinal pigment epithelium (RPE) atrophic area (B).

the RPE. This material is represented as a heterogenous reflective tissue between the RPE line and the inner segment-outer segment (IS/OS) photoreceptor junction line. It is often useful to magnify the SD-OCT image so as to verify that the RPE band is intact and that the serous retinal detachment observed corresponds to material resorption.
- Type 2 - multimodal imaging in eyes with AVL has identified a second distinct type of material within the subretinal space (see Figure $1 C$ and $D$ ). In these cases, there are no exudative signs on colour fundus photographs. FAF mostly shows hypofluorescent areas surrounded by some hyper-reflective zones. As in the first type of AVL, FA shows at the AVL site hypofluorescent areas surrounded by hyperfluorescent areas leaking with pin points in the late phases. ICGA also shows hypofluorescent areas in the early phases and hypo- and hyperfluorescent areas without leakage in the late phases.

On SD-OCT, there is always a typical appearance of type 2 AVL with pathognomonic abnormalities. These abnormalities are the imaging of the material resorption and the RPE hypertrophy reaction. Bruch's membrane is always visible with a hyper-reflective focal thickening or bumps of the RPE band just above it. There is always a hyporeflective 'empty' space between the thickened RPE and the IS/OS junction of the photoreceptors. This pseudo-serous detachment probably corresponds to the dysfunction of the RPE. This appearance is often associated with small dots that are presumed to be macrophages.

Serous retinal detachments with analogous features can be seen in angioid streaks and central serous chorioretinopathy (CSC). In pseudo-xanthoma elasticum, one can frequently observe this kind of 'vitelliform serous detachment' attributed to RPE dysfunction and not to CNV diffusion.

- Type 3 - (see Figure $1 E$ and F) type 3 AVL is less frequent, associated with pigment epithelial detachment (PED) and is mostly known as a non-neovascularised AMD. SD-OCT usually shows typical subretinal material with pseudo-serous detachment owing to RPE dysfunction. This serous detachment can be seen at the top of the PED or along the border of the detachment. FA and ICGA might be necessary to rule out occult CNV or polypoidal vasculopathy.

AVL can therefore be confused with CNV. SD-OCT and FAF are the key examinations to avoid such diagnostic mistakes.

\section{Pitfall 2 - Persistent Serous Detachment During} Anti-Vascular Endothelial Growth Factor Treatment There are two main pitfalls in the case of persistent serous detachment during anti-VEGF treatment - general RPE dysfunction and polypoidal vasculopathy.

- General RPE dysfunction. In some cases, after a period of active CNV treated with anti-VEGF injections, patients no longer complain of metamorphopsia. In biomicroscopy, no exudates or haemorrhages are present, but there is still a shallow serous detachment on SD-OCT.

Why do some cases present such a persistent retinal serous detachment? It is thought to be the result of dysfunction of the RPE pump. These cases need to be checked and retreated only if CNV signs recur.

Rarely, AMD is confused with chronic central serous chorioretinopathy (CSC; see Figure 2). FA and ICGA are necessary to arrive at the correct diagnosis. Enhanced depth imaging (EDI)-OCT allows for visualisation of increased choroidal thickness usually $>500 \mu \mathrm{m}$ in depth in CSC. ${ }^{16}$ 


\section{Figure 4: Outer Retinal Tubulations Observed with 'En-face' Spectral Domain Optical Coherence Tomography}

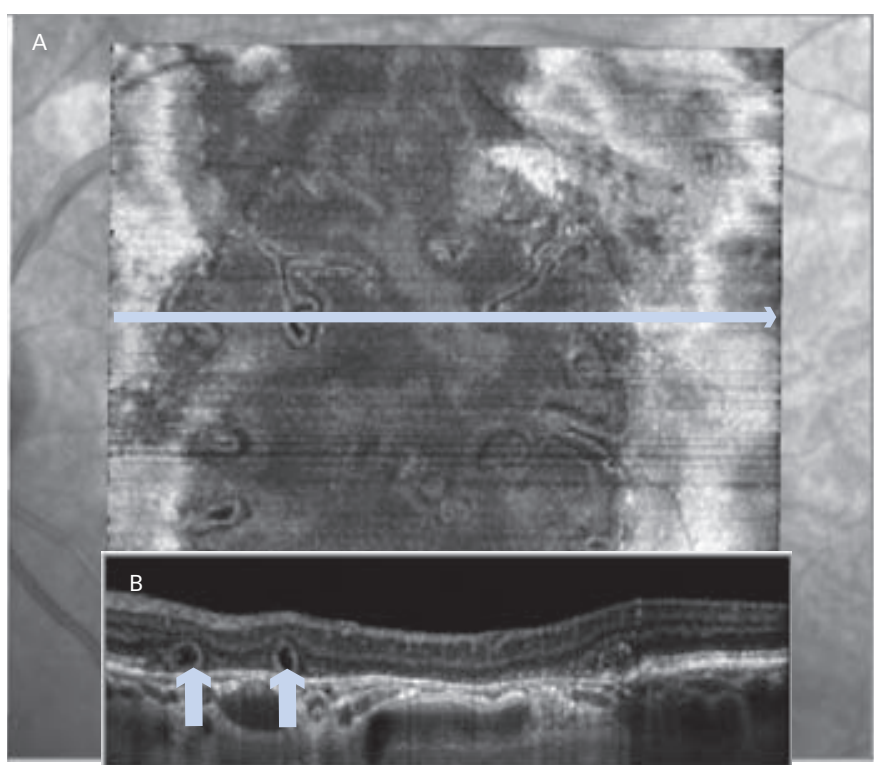

$A$ : the branching tubular structure of outer retinal tubulations.

$B$ : The corresponding B-scan demonstrates two lesions (arrows) bordered by a hyperreflective, well-defined rim located in the outer nuclear layer.

- Polypoidal vasculopathy. A false anti-VEGF resistance with persistent serous retinal detachment can occur in the case of polypoidal vasculopathy; this condition usually needs combination treatment for a better outcome.

It is particularly important to know that neovascular AMD that is refractory to a course of anti-VEGF monotherapy can harbour polypoidal choroidal vasculopathy. ${ }^{17}$ Polypoidal dilations might be suspected on SD-OCT, but ICGA is the most valuable tool for revealing polypoidal lesions. In this pathology, it is recommended to combine the angio-occlusion of the polyps using photodynamic therapy and the anti-permeability effect of anti-VEGF therapy on the branching vascular network.

\section{Pitfall 3 - Pseudocysts and Cystoid Cavities}

When a patient presents with intraretinal cysts during anti-VEGF treatment, the question is whether they are a sign of either exudation or degeneration? The cysts can be seen during routine AMD examination or during anti-VEGF treatment. Cohen et al. ${ }^{18}$ described hyporeflective cavities, located mainly in the internal nuclear layer but also in all other retinal layers, in dry AMD. He named these degenerative lesions 'retinal pseudocysts'. These lesions have no walls and differ from retinal oedema in lacking retinal thickening. They are always located near an area of RPE atrophy (see Figure 3). They have no angiographic signs and can be found in $27 \%$ of cases of geographical atrophy. They can also be seen during drusen resorption and are mostly found in the outer plexiform layer. The evolution of drusen has successive phases with first, an increase in drusen material and alteration of the surrounding tissues, followed by a disruption of the drusen, which is replaced by an atrophic area that can be correlated with pseudocysts.
The reason why such abnormalities develop is still not completely understood. Pseudocysts could correspond to Müller cell degeneration as seen in idiopathic macular telangiectasia type 2A and tamoxifen retinopathy. Anti-VEGF intravitreal injections have no effect on these cavities, which do not seem to be modified with time. Pseudocystic lesions, similar to those found in geographical atrophy, were described by Querques et al. ${ }^{19}$ in cases of exudative AMD in association with fibrovascular scarring. These hyporeflective, intraretinal lesions present with typically squared edges. These pseudocysts are often multiple and their location can be very variable. They can be found in the external nuclear or ganglion cell layer but are most frequently situated in the internal nuclear layer. Their degenerative character is confirmed by the absence of angiographic signs and their stability. They are seen in $39 \%$ of cases of exudative AMD complicated with a fibroglial scar. These pseudocystoid spaces probably correspond to retinal cell apoptosis. ${ }^{20}$

Another pitfall corresponds to CNV treated with anti-VEGF drugs and associated with epiretinal membrane. Sometimes, even if the CNV is closed, there is persistence of metamorphopsia associated with retinal thickening and pseudocysts. The diagnosis can be difficult and is based on the absence of improvement after anti-VEGF injection along with the absence of serous detachment and/or haemorrhages and exudates. This pitfall is more frequent in myopic eyes, where multiple pathologies are often associated.

\section{Pitfall 4 - Outer Retinal Tubulations}

Outer retinal tubulations (see Figure 4) can be seen in both atrophic and exudative AMD. On B-Scan SD-OCT, the outer tubulations are usually round, but they can also be elongated in shape, resembling a serous retinal detachment, in which case they have to be distinguished from CNV activity.

Zweifel et al. ${ }^{21}$ were the first to describe this aspect of retinal degeneration. Outer retinal tubulations represent a frequent finding as they are encountered in $56 \%$ of patients with exudative AMD and in $20 \%$ of cases of geographical atrophy. ${ }^{22}$ Outer retinal tubulations have no angiographic translation and do not change with time. 'En face' SD-OCT analysis shows the arborised network of interconnected tubules. Their size ranges from 60 to $600 \mu \mathrm{m}$. They are located in the outer nuclear layer, below the outer plexiform layer and over, or contiguous to, a fibrous and hyper-reflective thickening of the CC-RPE complex or over an atrophic area of the CC-RPE complex. These structures are optically empty, bordered by a mild but well-defined rim, and tiny punctuations are visible inside or along the inner face of the rim. The existence of the rim and the punctuations inside allow one to differentiate easily the outer retinal tubulations from an oedematous cavity. The outer retinal tubulations have no specific clinical or angiographic signs; the differential diagnosis is mostly with other intraretinal cystoid cavities most frequently found in the outer plexiform layer.

\section{Conclusion}

In conclusion, even if anti-VEGF treatments are based on SD-OCT data, the discussed pitfalls must be taken into consideration. An overall analysis of the patient's clinical examination, together with the imaging findings, is essential for accurate diagnosis and treatment of AMD. 
1. Mitchell, P, Smith, W, Attebo, K, Wang, JJ, Prevalence of agerelated maculopathy in Australia. The Blue Mountains Eye Study, Ophthalmology, 1995;102(10):1450-60.

2. VanNewkirk, MR, Nanjan, MB, Wang, JJ, et al., The prevalence of age-related maculopathy: the visual impairment project, Ophthalmology, 2000;107(8):1593-1600.

3. Klein, R, Klein, BE, Knudtson, MD, et al., Fifteen-year cumulative incidence of age-related macular degeneration: the Beaver Dam Eye Study, Ophthalmology, 2007:114(2):253-62.

4. Buch, H, Nielsen, NV, Vinding, T, et al., 14-year incidence, progression, and visual morbidity of age-related maculopathy: the Copenhagen City Eye Study, Ophthalmology. 2005;112(5):787-98.

5. Gariano, RF, Gardner, TW, Retinal angiogenesis in development and disease, Nature, 2005:438(7070):960-6.

6. Blaauwgeers, HG, Holtkamp, GM, Rutten, $\mathrm{H}$, et al., Polarized vascular endothelial growth factor secretion by human retinal pigment epithelium and localization of vascular endothelial growth factor receptors on the inner choriocapillaris. Evidence for a trophic paracrine relation, Am J Pathol, 1999;155(2):421-8.

7. Zarbin, MA, Rosenfeld, PJ, Pathway-based therapies for agerelated macular degeneration: an integrated survey of emerging treatment alternatives, Retina, 2010;30(9):1350-67.

8. Rosenfeld, PJ, Brown, DM, Heier, IS, et al, Ranibizumab for neovascular age-related macular degeneration, N Engl I Med, 2006;355(14):1419-31.

9. Brown, DM, Kaiser, PK, Michels, M, et al., Ranibizumab versus verteporfin for neovascular age-related macular degeneration, N Eng/ I Med, 2006:355(14):1432-44.

10. Gragoudas, ES, Adamis, AP, Cunningham, ET, Jr, et al., Pegaptanib for neovascular age-related macular

11. Brown, DM, Michels, M, Kaiser, PK, et al., Ranibizumab versus verteporfin photodynamic therapy for neovascula age-related macular degeneration: two-year results of the ANCHOR study, Ophthalmology, 2009;116(1):57-65.

12. Regillo, CD, Brown, DM, Abraham, $\mathrm{P}$, et al., Randomized, double-masked, sham-controlled trial of ranibizumab for neovascular age-related macular degeneration: PIER Study year 1, Am J Ophthalmol, 2008:145(2):239-48.

13. Kuiper, EJ, Hughes, JM, Van Geest, RJ, et al., Effect of VEGF-A on expression of profibrotic growth factor and extracellular matrix genes in the retina, Invest Ophthalmol Vis Sci. 2007:48(9):4267-76

14. Freund, KB, Laud, K, Lima, LH, et al., Acquired vitelliform lesions: correlation of clinical findings and multiple imaging analyses, Retina, 2011;31(1):13-25.

15. Finger, RP, Charbel Issa, $\mathrm{P}$, Kellner, U, et al., Spectral domain optical coherence tomography in adult-onset vitelliform macular dystrophy with cuticular drusen, Retina, 2010;30(9):1455-64

16. Imamura, Y, Fujiwara, T, Margolis, R, Spaide, RF, Enhanced depth imaging optical coherence tomography of the choroid in central serous chorioretinopathy, Retina,

2009;29(10):1469-73.

17. Stangos, AN, Gandhi, JS, Nair-Sahni, J, et al., Polypoidal choroidal vasculopathy masquerading as neovascular agerelated macular degeneration refractory to ranibizumab, Am . Ophthalmol, 2010;150(5):666-73.

18. Cohen, SY, Dubois, L, Nghiem-Buffet, S, et al., Retinal pseudocysts in age-related geographic atrophy, Am J Ophthalmol, 2010;150(2):211-7.

19. Querques, G, Zerbib, J, Santacroce, R, et al., The spectrum of subclinical Best vitelliform macular dystrophy in subjects with mutations in BEST1 gene, Invest Ophthalmol Vis Sci, 2011;52(7):4678-84.

20. Yannuzzi, LA, Bardal, AM, Freund, KB, et al., Idiopathic macular telangiectasia, Arch Ophthalmol, 2006;124(4):450-60

21. Zweifel, SA, Engelbert, $M$, Laud, $K$, et al., Outer retina tubulation: a novel optical coherence tomography finding, Arch Ophthalmol, 2009;127(12):1596-1602.

22. Wolff, B, Maftouhi, MQ, Mateo-Montoya, A, et al., Outer retinal cysts in age-related macular degeneration, Acta Ophthalmol, 2011;89(6):e496-9. 